\title{
Passé, présent, futur : au-delà des frontières ? \\ Un temps simultané dans le roman graphique From Hell d'Alan Moore
}

François Duclos, Université de Nantes

Les cultures occidentales ont l'habitude de considérer le temps comme une trinité inébranlable : passé, présent et futur. On retrouve ce séquençage dans le sens de lecture d'un livre et, de manière plus visible encore, dans une bande dessinée : l'illusion du temps qui passe est rendue par le séquençage des vignettes dans l'espace du livre. Le temps donc ne fait qu'un avec l'espace dans la bande dessinée, contrairement à d'autres médias comme le cinéma où c'est la succession des images sur un même espace (l'écran) qui transcrit l'illusion du temps (McCloud 94). Cet article vise à s'interroger sur la représentation du temps en bande dessinée, et comment ce médium, par sa forme hybride, permet de suggérer une conception de temps simultané. Pour ce faire, une étude narratologique permet de disséquer les pages du corpus choisi afin de chercher les éléments qui renvoient à cette représentation. Le roman graphique ${ }^{1}$ From Hell, créé par Alan Moore et Eddie Campbell, propose une réécriture du mythe de Jack l'Éventreur, figure majeure de l'imaginaire collectif occidental. Les meurtres de cinq prostituées qui ont fait frémir la ville de Londres à l'automne 1888 restent encore aujourd'hui un mystère pour beaucoup de spécialistes. Le roman graphique ne porte pas tant sur l'affaire de l'Éventreur que sur l'espace dans lequel le phénomène s'est produit puisque l'identité de l'Éventreur est révélée au lecteur dès le deuxième chapitre du récit. Les auteurs s'intéressent à l'analyse du phénomène humain du meurtre ainsi que sa signification dans l'Histoire et dans l'espace londonien. Si la première lecture du roman nous offre une fresque monumentale du Londres victorien, avec toutes ses réalités sociales et politiques (abondamment documentées), une relecture avisée nous dévoile un intérêt plus large : une réflexion sur le temps. Le récit n'est pas seulement centré sur cette période de fin de siècle décadent, mais l'analyse pour la mettre en perspective sur une échelle historiographique bien plus vaste. Ce phénomène social de 1888 est-il unique en soi ou n'est-ce qu'une résonance de l'Histoire qui se répète ? Plus importante que le personnage du tueur en série, la ville devient au fil du récit le personnage principal de cette histoire, dans toutes ses strates, recouvrant du poids de l'Histoire ses habitants. À travers le récit, les barrières temporelles sont transgressées pour mettre en avant une représentation d'un temps simultané, dans lequel on peut évoluer dans plusieurs directions. Quelles sont les possibilités propres à la bande dessinée ? Qu'est-ce que le médium peut apporter à la réflexion sur le temps? Nous nous pencherons d'abord sur 
l'espace textuel et son rapport à l'image, qui déconstruit la conception linéaire du temps. Nous verrons ensuite comment l'espace visuel du livre en tant qu'objet permet de représenter un temps simultané, avant de plonger dans un espace plus abstrait, celui de l'ésotérisme.

\section{L'espace textuel révèle un temps multicouche}

L'espace textuel est le premier qui peut aiguiller le lecteur dans la réflexion sur le temps proposée par les auteurs. C'est l'élément le plus explicite qui expose le temps comme problématique centrale du roman graphique. Si nous nous intéressons à la structure du roman graphique, on s'aperçoit qu'il est difficile pour le lecteur de trouver un temps de référence précis au récit. Le point focal est l'année 1888, date des agissements du meurtrier londonien. Mais le récit se développe dans d'autres époques, par l'utilisation d'analepses et de prolepses. Les quatorze chapitres qui constituent le récit de l'Éventreur sont encadrés par un prologue et un épilogue dans lesquels on suit Abberline, inspecteur chargé de l'enquête durant les événements, et son ami Lees, sur une plage de Bournemouth en 1923. À travers le récit, de nombreuses analepses présentent au lecteur des événements bien antérieurs à 1888, élargissant ainsi le spectre temporel de l'histoire. Non seulement les auteurs présentent des scènes antérieures ou postérieures à 1888, mais ils rendent encore plus poreuses les frontières entre passé et futur en mélangeant analepses et prolepses. Un des meilleurs exemples pour illustrer ce mélange est sans doute le chapitre deux, structuré comme la matrice de tout le récit : tous les thèmes et toutes les problématiques abordés sont concentrés dans ce chapitre. Le lecteur y découvre le personnage de William Gull à différents épisodes de sa vie dans des scènes fragmentées sans transitions apparentes. La première page illustre parfaitement l'atemporalité qui sera développée à travers tout le récit. Les cases sont entièrement noires et ne représentent aucun lieu, aucun espace et donc aucun temps précis. Les phylactères ${ }^{2}$ que l'on peut lire n'ont aucun lien entre eux et sont incompréhensibles pour le lecteur, du moins au moment de la lecture de cette page seulement. En effet, plus tard dans le chapitre, ces phylactères seront repris et remis dans le contexte de leurs scènes respectives. Le lecteur est confronté plusieurs fois au fil du récit à des scènes qui ne peuvent être placées ou classifiées selon un unique axe temporel. Dans cette première page, nous trouvons déjà une contradiction au niveau intradiégiétique. Comme nous l'avons vu, par rapport à la période largement couverte dans le roman, l'automne 1888, cette page est une analepse ; les phylactères présents viennent de scènes antérieures aux événements principaux. Le cartouche de la page, indicateur de lieu et de temps, place cette page en juillet 1827. Les phylactères de cette page ne correspondent pourtant pas à l'année 1827 : comme le lecteur le voit à la lecture du chapitre, ces phylactères viennent de dialogues 
à d'autres moments de la vie de Gull ; ils sont donc des prolepses par rapport à la chronologie du récit. Pourquoi alors indiquer la date et le lieu à la première page alors que ces données correspondent à la scène de la deuxième page ? Voilà la technique utilisée par les auteurs pour déconstruire la structure du temps. Si les phylactères ne peuvent logiquement pas être présents dans l'espace de 1827, leur inclusion ici revient à briser les frontières du temps. Leur présence physique dans l'espace de 1827 est confirmée à la page suivante lorsque le chaos d'obscurité de la première page se transforme en tunnel où l'on retrouve William Gull à onze ans avec son père sur leur barge. William, déjà sensible au monde invisible, explique à son père : «I was listening to the echoes in the tunnel » $(\mathrm{ch} .2,2)^{3}$. La première page offre ainsi une impression de déjà-vu au lecteur en lui présentant des bribes de conversations qu'il retrouvera plus tard dans leur entièreté. En ce qui concerne le personnage de William, rien n'est affirmé dans cette scène mais seulement suggéré, ce qui laisse le choix d'interprétation au lecteur. William a-t-il, lui aussi, une impression de déjà-vu en entendant des bribes de son futur ? Ou le chaos de la première page n'est-il destiné qu'au lecteur?

Cet effet d'atemporalité rendu par les analepses et prolepses est renforcé par la juxtaposition des cases. Le fait que les transitions entre les scènes soient abruptes, donne un effet de conglomérat : elles sont présentées au lecteur comme des bribes, des souvenirs qui n'ont pas de hiérarchie et qui ont tous la même valeur, qu'ils soient anciens ou récents. Alors qu'elles sont complètement dissociées dans le temps, ces scènes sont ici mises côte à côte, comme si elles présentaient une séquence logique. Au niveau extradiégétique, l'intertextualité est un important facteur d'atemporalité. Moore utilise très souvent des références intertextuelles, créant un dialogue implicite avec le lecteur. Intégrer des références à des œuvres réalisées avant mais aussi après le temps du récit brouille encore une fois les limites du temps de référence pour le lecteur. Les épigraphes au début de chaque chapitre offrent une piste de lecture sur les événements du chapitre pour nourrir la réflexion sur le temps. Ces références intertextuelles se retrouvent aussi dans l'espace intradiégétique. Dans l'histoire, Moore amène le personnage du docteur Gull à rencontrer Joseph Merrick, un homme devenu célèbre souffrant vraisemblablement du syndrome de Protée, à l'origine de remarquables déformations de son squelette. Cette rencontre, si elle est plausible, n'est pas vérifiée historiquement. Le personnage de Joseph Merrick, ainsi que la chambre dans laquelle il loge, sont représentés par Campbell exactement comme on peut les voir dans le film de David Lynch, Elephant Man (1980). Outre les références intertextuelles qui se fondent dans le décor de ce Londres décadent, d'autres intertextes viennent explicitement interroger la temporalité du récit. Dans une autre scène du chapitre deux, Gull se promène dans le quartier de Whitechapel avec son ami et mentor Hinton. 
Leur discussion les amène à parler de l'essai écrit par le fils d'Hinton ${ }^{4}$ : «They suggest Time is a human illusion [...] That all times coexist in the stupendous whole of eternity ${ }^{5}$ (ch. 2 , 12). Ces références intertextuelles font du lecteur un agent actif du sens du récit. L'intertextualité brouille la frontière entre fiction et réalité dans From Hell, et montre au lecteur que les faits présentés importent peu, c'est leur signification que l'on doit dégager à l'aide des indices disséminés par les auteurs. Plutôt que d'apporter une énième théorie sur le mystère qui entoure Jack l'Éventreur, les auteurs utilisent ce phénomène social pour proposer au lecteur une réflexion sur l'historiographie.

\section{Un temps simultané dans l'espace visuel}

Le roman traditionnel est unimodal : les mots et les phrases induisent une progression de lecture linéaire qui permet à l'auteur de garder le contrôle de la progression du lecteur. La bande dessinée combine texte et image. Cette multimodalité partage ainsi des caractéristiques avec l'art pictural, le roman et le cinéma. Will Eisner, l'un des pionniers de l'art de la bande dessinée tel que nous le connaissons aujourd'hui interroge la forme du médium dans Comics and Sequential Art. Ce qui différencie la bande dessinée des autres supports auxquels elle emprunte des caractéristiques, c'est la séquentialité qui intervient au cœur de la temporalité. Cette caractéristique de format permet de concevoir le temps d'une manière particulière qui correspond bien à l'objectif narratif de Moore : représenter l'espace-temps. Comme l'explique Scott McCloud, dans la bande dessinée, c'est l'espace qui permet de séparer temporellement une image d'une autre : «Time and space are one and the same $»^{6}(100)$. Dans From Hell, l'agencement des pages est très régulier et conventionnel selon la terminologie de Benoît Peeters. ${ }^{7}$ La plupart des pages sont découpées de la même manière ; trois bandes de trois cases de même taille. Ce séquençage régulier suggère un passage du temps constant et neutre, non manipulé par les auteurs. Cette composition souligne l'aspect historique et réaliste du récit. Toujours selon Peeters, cette utilisation conventionnelle de la page permet de mettre en avant le récit. Cependant, cette régularité dans la composition peut être utilisée ou même brisée pour modifier la perception du lecteur et ainsi proposer un regard critique sur le récit. On a alors affaire à un usage rhétorique, où la composition n'impose plus son cadre au récit mais où au contraire, c'est l'action qui impose la forme et la position des cases pour obtenir un effet de style (Peeters 52).

Cette utilisation prend donc différentes formes et remplit différentes fonctions. Surtout, chaque changement apporte une perturbation dans la perception du temps. Ainsi, après avoir tué la deuxième prostituée sur sa liste, Gull et son acolyte Netley laissent le corps de la pauvre 
femme sur le trottoir en pleine nuit. Une série de trente-deux vignettes se succèdent, ayant toutes la même composition. On voit le corps de loin, sur le trottoir dans la rue déserte. Cet étirement suggère la longue période de temps qui s'écoule avant que le corps ne soit découvert. En gardant le format de page constant mais en multipliant les cases similaires, les auteurs ralentissent la représentation du temps. Le lecteur se retrouve dans une position de voyeur, forcé de regarder alors qu'il est incapable d'agir.

Des cases sans cadre viennent également briser la régularité de la composition. Cette différence de forme incite le lecteur à marquer une pause dans sa lecture et à s'attarder sur la vignette concernée. La première hallucination à laquelle Gull est sujet, est représentée dans un splash, c'est-à-dire une image qui prend tout l'espace de la page et qui n'est pas restreinte par un cadre. Lors de cette hallucination, Gull voit les trois figures de la divinité franc-maçonnique Jah-Bul-On, entité titanesque qui occupe tout le ciel de l'image. L'absence de cadre fait donc sortir l'image de la progression régulière du récit et souligne l'atemporalité liée au caractère éternel des divinités : "Time is no longer contained by the familiar icon of the 'closed' panel but instead haemorrhages and escapes timeless space. Such images can set an atmosphere or evoke a kind of place that endures over the whole scene timelessly» (McCloud 103) ${ }^{8}$. Les auteurs mettent donc en place différentes techniques pour modeler le temps à leur façon à travers l'utilisation des cases ou vignettes. Mais l'agent (invisible) principal du temps dans la bande dessinée reste l'espace intericonique : ces lignes blanches qui courent entre toutes les cases d'une page et que l'on nomme vulgairement les gouttières. Si les cases forment une séquence qui divise le temps et l'espace, l'espace intericonique est ce qui sépare les cases, une sorte d'antimatière de la bande dessinée qui définit un espace atemporel. Ce dernier permet de créer le séquençage, fondamental dans la plupart des bandes dessinées. Cet espace représente l'ellipse temporelle entre chaque vignette et permet de rendre la séquence cohérente pour le lecteur en laissant à son imagination le soin de remplir cette ellipse. C'est le système de «closure » (que l'on pourrait traduire par système elliptique), présenté par McCloud comme la dimension la plus importante de la bande dessinée. Le temps se retrouve donc compressé entre chaque case, ne demandant qu'à s'étendre. Cet espace, souvent de même taille entre chaque case, peut représenter une ellipse de temps variée à chaque fois. Cette séparation des cases, si elle n'est pas toujours utilisée par les auteurs (Eisner fait des pages sans vignettes cadrées), donne au lecteur une vision globale de la progression du récit. D'un seul coup d'œil, nous voyons le présent, le passé et le futur. La forme même du médium permet donc aux auteurs de suggérer un temps simultané.

Le travail d'Eddie Campbell participe à la réflexion sur le temps qui s'opère tout au 
long du roman. Le style impressionniste de Campbell rappelle les gravures du dix-neuvième siècle : son trait au crayon est prononcé, la profondeur et les volumes sont rendus sur le support en deux dimensions de la page par les hachures et le noir et blanc produit souvent un effet de clair-obscur saisissant. Campbell inclut de nombreuses fois dans ses illustrations des indicateurs de temps, horloges en arrière-plan, calendrier sur le bureau d'Abberline, autant de marqueurs de temps qui suggèrent que les policiers ne sont pas seulement en train de courir après un meurtrier, mais après la progression effrénée de l'histoire et du temps elle-même. Dans le récit, Gull considère que ses actes aboutissent à l'entrée de Londres dans le vingtième siècle. Par ses actions inhumaines, il contribue selon lui au progrès de la cité. Le poids de l'Histoire semble peser sur les personnages qui sont incapables de se libérer des chaînes du temps qui passe. Contrairement à Gull qui est capable de se défaire de ces chaînes, les autres personnages sont prisonniers de l'époque dans laquelle ils vivent. Gull est le seul à être lucide et à percevoir l'architecture de l'Histoire qui se déroule dans les rues de Londres. De la même manière qu'il perçoit des bribes d'autres époques et que sa perception du temps s'en trouve modifiée, le style graphique de Campbell trouble également les frontières entre premier et arrière plans, personnages et monuments, êtres du présent et fantômes du passé.

Nous l'avons vu plus tôt, les auteurs présentent au lecteur des scènes qui ne se sont pas encore passées pour rendre une impression de déjà-vu. Mais ils mettent également à profit le système elliptique afin de donner au lecteur le même pouvoir que Gull : percevoir des bribes du futur. En effet, la juxtaposition de vignettes incite le lecteur à construire une certaine hypothèse sur la suite du récit par association d'idées. De nombreuses transitions entre scènes offrent ainsi des indices implicites sur l'histoire, suggérant le poids inéluctable du temps qui passe. Par exemple, une vignette représentant une des prostituées dans son lit, à moitié nue et une bouteille d'alcool à la main est suivie par une reproduction du tableau de William Hogarth The Reward of Cruelty. Cette lithographie représente une séance de dissection d'un corps. La position du corps de la femme est étonnamment similaire à la position du corps entre les mains des scientifiques, tandis que son ventre ouvert laissant voir ses entrailles amène le lecteur à imaginer ce qui attend la jeune femme puisqu'elle est sur la liste de l'Éventreur. Un lecteur avisé qui se serait renseigné sur l'affaire de l'Éventreur aurait accès à toutes les informations historiques nécessaires pour connaître l'issue du récit. Malgré certaines retouches de l'Histoire de la part des auteurs, les faits présentés sont historiquement exacts selon l'hypothèse de Knight choisie pour créer le récit. Le lecteur, avant même de commencer à lire From Hell, peut déjà savoir les grandes lignes de ce qu'il va lire. Rien ne lui est caché et il est donc capable d'avoir accès à la fois au passé et au présent du récit, mais aussi à son futur. Et pour la plupart des 
lecteurs qui n'auraient pas les informations concernant l'affaire, des indices comme celui mentionné plus haut leur permettent d'anticiper ce qui va suivre. Ainsi le lecteur se retrouve en quelque sorte dans la même position que l'Éventreur du récit; tous deux ont la capacité de percevoir le futur.

Toutes ces techniques de déconstruction du temps et de superposition du passé, du présent et du futur tendent naturellement vers un point d'orgue dans le récit. William Gull voit dans les meurtres qu'il perpétue une œuvre monumentale, une mission divine qui lui donnera accès à une connaissance supérieure synonyme de progrès pour le reste de l'humanité. Les meurtres violents qu'il accomplit le plongent dans une ivresse sauvage et surnaturelle, une transe qui le rend conscient d'une autre facette de la réalité. Les meurtres sont à chaque fois plus barbares que les précédents à mesure que les hallucinations de Gull prennent le pas sur sa perception du réel. Ainsi, Gull progresse vers une sorte de révélation lui donnant accès à une nouvelle dimension de l'espace-temps, ultime étape du parcours de l'Éventreur. Le dernier meurtre qu'il accomplit, est le point culminant du récit. Le temps narratif est dilaté, chaque action étant ralentie et présentée dans une longue suite de vignettes. Lentement, Gull découpe des morceaux de chair de sa victime et cet acte inhumain devient pour lui un instant sacré, un rituel qui lui permet de s'élever au-delà du monde tridimensionnel perçu par le commun des mortels. Alors qu'il travaille méticuleusement dans la chambre de la victime, les fantômes du passé apparaissent et Gull se retrouve des années en arrière, à donner un cours d'anatomie dans une salle d'université (ch. 10,14). Très naturellement, Gull fait sa lecture aux silhouettes spectrales qui l'entourent. Quelques instants plus tard, il se retrouve au milieu de bureaux modernes, avec des employés travaillant sur ordinateur, discutant au téléphone portable. Devant l'indifférence des employés qui ne perçoivent sans doute pas la présence de cet être du passé, Gull se désespère de l'état de la vie moderne et se rend compte qu'il n'a pas encore atteint le dernier stade de son ascension. Celle-ci ne sera complète qu'au moment de sa propre mort, au dernier chapitre du récit.

\section{La philosophie occulte comme interprétation du temps}

L'étude de l'espace textuel et visuel de From Hell nous a permis d'identifier les mécanismes qui permettent de rendre explicite pour le lecteur la conception d'un temps simultané. Mais pour mieux comprendre cette conception, il est nécessaire de s'intéresser à un autre aspect central du roman graphique et de l'œuvre de Moore en général : l'ésotérisme. Cette philosophie occulte est présentée dans le roman comme un domaine d'étude légitime, qui permet d'explorer des domaines que la science ne permet pas d'expliquer. Dans son Histoire 
de la philosophie occulte, Sarane Alexandrian décrit ce domaine non pas comme un sujet d'obscurantisme mais bien comme un complément utile et indispensable aux autres sciences, immanent à la psyché humaine :

Au lieu donc de considérer ces croyances magiques comme lettres mortes, on doit y voir les signes vivants d'un état d'esprit en perpétuelle évolution au cours des âges. Elles correspondent à des ressources psychiques permanentes de l'humanité que la philosophie occulte prétend définir, augmenter, utiliser pour le mieux de l'individu, ce qui rend son étude indispensable, parmi d'autres, à une bonne appréciation du devenir de l'être humain. (14)

La frontière entre visible et invisible n'est jamais claire dans le roman graphique et suggère la présence de forces inconnues et supérieures. La ville de Londres est placée au cœur de cette tension et devient peu à peu un personnage central et oppressant pour tous ses habitants. Les superstitions et les nombreuses références à des légendes urbaines témoignent du poids du surnaturel sur le conscient des habitants. L'ambivalence entre pragmatisme et occultisme est constamment nourrie à travers le récit et amène le lecteur à ne plus savoir que croire, ou bien à faire un choix dans ce qu'il souhaite croire.

Lees est le personnage qui illustre le mieux cette ambivalence. Conseiller surnaturel de Sa Majesté lors des évènements de 1888, Lees gagne sa vie comme médium entre le monde matériel et l'au-delà, notamment en donnant à la Reine des nouvelles de son défunt mari par séances d'invocations. Méprisé par Gull qui le considère comme un charlatan, c'est néanmoins Lees qui amènera l'inspecteur Abberline à découvrir le véritable meurtrier en le convainquant que des visions lui ont révélé l'identité du tueur. Dans le prologue cependant, Lees avoue à Abberline que ces visions n'étaient qu'une supercherie : «All of them. All the visions. I made them up » (prologue, 3$)^{9}$. Le surnaturel ne s'impose jamais clairement dans le récit, mais suggère au lecteur la possibilité d'autres facettes de la réalité que la plupart d'entre nous ne percevons pas. Lees n'est-il effectivement qu'un charlatan ou a-t-il de réels pouvoirs psychiques? Gull le traite avec condescendance et se considère comme le seul digne d'un savoir supérieur. En même temps que Gull dévoile de son plein gré son identité à l'inspecteur Abberline, il murmure à l'oreille de Lees effrayé : «Tell me, Mr. Lees : have you ever truly had a vision? A real vision? No? I didn't think so... But I have » $\left(\right.$ ch. 12, 12) ${ }^{10}$. En se livrant entièrement aux deux hommes, Gull affirme son pouvoir, qu'il soit de nature occulte ou simplement persuasive. Cet aveu délibéré ne mènera d'ailleurs pas à son arrestation, Abberline n'ayant pas quant à lui le pouvoir nécessaire pour traiter l'affaire avec un personnage si important.

Dans le récit, un parallèle explicite est établi entre le personnage de Gull et William 
Blake, qui apparaît d'ailleurs dans le roman graphique. Artiste adepte de philosophies occultes, Blake a été de son vivant hautement controversé : « Shall I call him Artist or Genius - or Mystic or madman ? Probably he is all $»^{11}$, écrit son biographe Henry C. Robinson (Symons 253). Blake est une source d'inspiration primordiale pour Moore; certains articles s'intéressent particulièrement aux parallèles qui lient les deux auteurs. Pour le lecteur, Gull apparaît aussi ambivalent que le poète. Est-il complètement fou, ou est-ce un visionnaire lucide, un génie ? C'est bien au dernier chapitre que Gull s'élève dans une dimension qu'il croit supérieure. Ayant été envoyé à l'asile par ses supérieurs franc-maçonniques qui le considéraient déviant et dangereux, le docteur Gull s'éteint dans sa cellule. Alors que son esprit quitte son corps mourant, Gull est enfin libéré de toute contrainte spatio-temporelle. Il traverse alors des multitudes d'épisodes de sa propre vie et d'autres époques. Le lecteur retrouve des vignettes, voire des pages entières qu'il a déjà lues dans les chapitres précédents. Faisant appel à la représentation bien connue selon laquelle la vie d'un individu défile devant ses yeux lors de sa mort, les dessins présents reprennent des scènes du passé, alors que les phylactères apportent un nouveau commentaire et une perception différente de ces évènements. Gull atteint un nouvel état de conscience qui lui permet de se mouvoir dans d'autres espaces temporels. On peut alors revoir la discussion entre Gull et Hinton à propos de l'essai «What Is the Fourth Dimension ? » dont la portée est ici développée. Les actes de Gull apparaissent alors comme un leitmotiv de l'Histoire, où l'esprit de l'Éventreur, diffus dans des personnages de différentes époques, réitère régulièrement ce genre d'évènements au cours des âges. Et Gull devient cette Histoire, il devient lui-même l'espace-temps : «Movement, and yet there is no movement. There is not space. There is not time, and therefore nothing moves, but only is $»(\text { ch. 14, 14) })^{12}$. Son esprit ainsi que ce qu'il représente dans la psyché collective est diffus dans l'Histoire humaine : «I am set free from flesh and time. I am become a symbol in the human soul; a fearful star in mankind's inner firmament. And still I rise. Eternity itself shall not contain me » $(\mathrm{ch} .14,14)^{13}$. Durant toute son ascension, il prend conscience de ce qu'il devient, une entité immatérielle qui contient tout, passé, présent et futur. C'est à la fin de son ascension que Gull quitte définitivement le monde terrestre, pour accéder à d'autres sphères au-delà de la compréhension. Cette ascension est illustrée par une page sans aucune case, aucun cadre, aucun phylactère. L'espace de la page s'étend à l'infini. Gull s'évapore au-delà du temps, laissant derrière lui une trace, une phrase incomplète, infinie : « and then I... » $(\mathrm{ch} .14,28)^{14}$. 


\section{Conclusion}

Tous ces éléments font de From Hell une introduction subtile et complexe à la conception du temps mais aussi à l'univers de Moore. Il s'inscrit dans la pensée philosophique de l'éternalisme inspirée par la théorie de la relativité où le temps est considéré comme une dimension de l'espace. Le format du roman graphique semble être un support parfait pour déconstruire le séquençage illusoire du temps et en proposer une conception simultanée. La mécanique centrale du médium, en nous faisant percevoir le temps à travers l'espace de la page, soutient évidemment cette conception éternaliste. Moore s'inscrit également dans le mouvement littéraire récent en Grande-Bretagne que Peter Ackroyd a appelé «Transitional Writing ${ }^{15}$; des récits qui proposent une relecture du passé en mélangeant faits et fiction et nous invitent à réinterpréter le passé à travers le prisme du présent pour construire dès aujourd'hui le futur. Le récit From Hell offre au lecteur une expérience singulière qui amène à réfléchir sur la nature ontologique du temps et nous interroge sur l'historiographie, la folie, et notre perception infime du monde.

\section{Ouvrages cités}

Alexandrian, Sarane. Histoire de la philosophie occulte. Paris : Payot, 1994.

Allen, Graham. Intertextuality. New York : Routledge, 2000.

Baetens, Jan et Hugo Frey. The Graphic Novel : An Introduction. New York : Cambridge UP, 2015.

Eisner, Will. Comics and Sequential Art. New York : Norton, 2008 [1985].

McCloud, Scott. Understanding Comics : The Invisible Art. New York : Harper Collins, 1994. Moore, Alan et Eddie Campbell. From Hell. London : Knockabout, 2014 [2000].

Peeters, Benoît. «Quatre conceptions de la page ». Case, planche, récit : lire la bande dessinée. Paris : Casterman, 1998. 41-62.

Symons, Arthur. William Blake. New York : Dutton, 1907.

Vianu, Lidia. «The Mind is the Soul ». Entretien avec Peter Ackroyd. Desperado EssayInterviews. Bucarest : Bucharest UP, 2006.

\footnotetext{
Notes

${ }^{1}$ Le roman graphique est un terme apparu à la fin des années 1970 et désigne des bandes dessinées qui, contrairement aux séries, ont une unité narrative (un début, un milieu et une fin) et qui expérimentent avec la forme du médium. Certains auteurs ont proposé ce terme pour changer la vision de la bande dessinée qui jusqu'alors était considérée comme un médium indigne d'intérêt littéraire et réservé aux adolescents (Baetens et Hugo)

${ }^{2}$ Les phylactères, aussi appelés bulles, sont l'espace dans lequel sont contenues les paroles et/ou les pensées des personnages.

${ }^{3}$ Je traduis : « J'écoutais l'écho du tunnel ».
} 


\footnotetext{
${ }^{4}$ Charles Howard Hinton est un mathématicien et philosophe anglais qui a travaillé sur l'idée d'une quatrième dimension de l'espace, notamment dans son essai «What is the Fourth Dimension? » publié dans Scientific Romances en 1884.

${ }^{5}$ Je traduis : «Ils suggèrent que le Temps est une illusion humaine [...] Que toutes les temporalités coexistent dans le tout prodigieux de l'éternité ».

${ }^{6}$ Je traduis : « le temps et l'espace ne font qu'un ».

${ }^{7}$ Benoît Peeters est auteur de bandes dessinées mais aussi d'essais critiques sur le médium. Il est notamment spécialiste d'Hergé et de l'univers de Tintin et est auteur, en collaboration avec François Schuiten de la série Les cités obscures.

${ }^{8}$ Je traduis : «Le temps n'est plus contenu dans l'icône familière de la vignette fermée, mais à la place se répand et s'échappe dans un espace hors du temps. De telles images peuvent créer une atmosphère ou évoquer un type de lieu qui perdure durant toute la scène de manière atemporelle ».

${ }^{9}$ Je traduis : «Toutes. Toutes ces visions. Je les ai inventées ».

${ }^{10}$ Je traduis : «Dites-moi M. Lees, vous est-il déjà vraiment arrivé d'avoir une vision ? Une véritable vision ? Non ? C'est bien ce que je pensais... Moi, en revanche, j'en ai eu ».

${ }^{11}$ Je traduis : «Devrais-je l'appeler Artiste ou Génie - Mystique ou fou ? Il est probablement tout cela ».

${ }^{12}$ Je traduis : « Mouvement, et pourtant il n'y a pas de mouvement. Il n'y a pas d'espace. Il n'y a pas de temps, et par conséquent rien ne se déplace, mais simplement existe ».

${ }^{13}$ Je traduis : «Je suis libéré de la chair et du temps. Je suis devenu un symbole dans l'âme de l'Homme ; une étoile effrayée dans le firmament intérieur de l'humanité. Et je m'élève encore. L'Éternité elle-même ne saurait me retenir ».

${ }^{14}$ Je traduis : « et enfin je... ».

15 D'après son entretien avec Lidia Vianu.
} 